\title{
Omega-3 polyunsaturated fatty acids and the inflammatory state of the Caco-2 gut epithelium model
}

\section{Abstract}

The gut epithelium is a protective interface between the external environment and the human body. This epithelium interacts with a multitude of internal stimuli from the bloodstream and immune cells, and luminal stimuli from microorganisms and the diet. Disruptions to the epithelium are seen in inflammatory bowel diseases and coeliac disease. The human adenocarcinoma cell line (Caco-2) is an in vitro model used to assess the interactions between nutrients and gut epithelium. Long-chain omega-3 (n-3) polyunsaturated fatty acids (PUFAs), such as eicosapentaenoic acid (EPA) and docosahexaenoic acid (DHA), have anti-inflammatory effects via the production of anti-inflammatory eicosanoids, interactions with immune cells and reductions in pro-inflammatory cytokines and chemokines. The aim of this study is to assess the anti-inflammatory properties of DHA and EPA in stimulated Caco-2 monolayers. Caco-2 cells were seeded at 70,000 cells $/ \mathrm{cm}^{2}$ and grown to confluence before being allowed to fully differentiate (approx. 21 days total). Cytokines (TNF- $\alpha$, IFN- $\gamma$, and IL-1 $\beta$ ) and peptic-tryptic (PT-) gliadin were used as inflammatory stimulants. EPA and DHA incubations occurred 48 hours pre-stimulation. Tight junction function and morphology was determined using transepithelial electrical resistance measurements and confocal microscopy. Inflammatory markers, including IL-6, IL-8, and IL-17, were assessed by multiplex. Stimulatory cytokines induced tight junction dysfunction and increased pro-inflammatory mediator production in Caco-2 cells. PT-gliadin, DHA and EPA treatment did not alter paracellular permeability or stimulant-induced production of pro-inflammatory mediators. Further investigation of the inflammatory role of n-3 PUFAs and PT-gliadin in the Caco-2 model is required. Future work will assess the composition of PT-gliadin by electrophoresis and whether co-incubation of n-3 PUFAs and inflammatory cytokines will alter paracellular permeability and mediator output of Caco- 2 cells.

\section{Conflict of Interest}

There is no conflict of interest 